\title{
Decision on the number of turns in the eRHIC Nov'15 design
}

\author{
S. Brooks
}

\section{Collider-Accelerator Department Brookhaven National Laboratory Upton, NY 11973}

\author{
U.S. Department of Energy \\ Office of Science, Office of Nuclear Physics
}

Notice: This document has been authorized by employees of Brookhaven Science Associates, LLC under Contract No. DE-SC0012704 with the U.S. Department of Energy. The United States Government retains a nonexclusive, paid-up, irrevocable, world-wide license to publish or reproduce the published form of this document, or allow others to do so, for United States Government purposes. 


\section{Decision on the Number of Turns in the eRHIC Nov'15 Design}

Stephen Brooks, 2015-Dec-01

\section{Introduction}

When moving from the "Jun'15" to the "Nov'15" eRHIC FFAG design, the number of accelerating passes through the linac was reduced from 16 to 12 . There are an equal number of decelerating passes, so the total reduced from 32 to 24 . At the same time, the linac energy was increased from $1.322 \mathrm{GeV}$ to $1.665 \mathrm{GeV}$ and the RF frequency changed from $422 \mathrm{MHz}$ to $647 \mathrm{MHz}$. The maximum beam energy remained approximately constant, changing from $21.164 \mathrm{GeV}$ to exactly $20 \mathrm{GeV}$.

The RF frequency choice is discussed in another eRHIC note. This document describes the trade-offs and limitations affecting the number of turns in eRHIC, while keeping the maximum energy at approximately $20 \mathrm{GeV}$.

\section{Factors Influencing Number of Accelerating Turns}

\subsection{Linac Length}

Assuming the maximum energy of eRHIC remains fixed at $20 \mathrm{GeV}$ and that the injector energy remains small compared to the main linac, the energy gain required of the linac is $20 / \mathrm{N} \mathrm{GeV}$ where $\mathrm{N}$ is the number of accelerating turns. The previous design had a $1.322 \mathrm{GeV}$ linac with a total installed length of $123.55 \mathrm{~m}$. The linac length becomes a constraint when it exceeds the available space in the two o'clock straight of the RHIC tunnel (Figure 1). The only way to have more energy gain per turn is to have another linac installation in a different straight section of RHIC, which would incur the additional costs of two more splitters/combiners and cryogenic infrastructure at the new location $\sim 500$ m away.

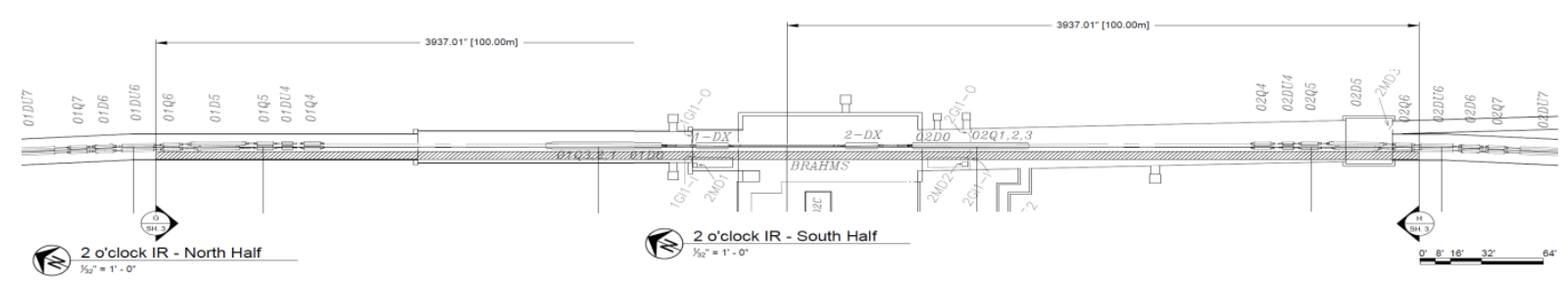

Figure 1: The $200 \mathrm{~m}$ of straight space available in the RHIC two o'clock straight for the eRHIC linac.

In fact, longitudinal space in this straight will become a constraint before the linac reaches $200 \mathrm{~m}$ in length because the following items also have to occupy the straight space:

- The dipole merging the $\sim 20 \mathrm{MeV}$ injection line, before the linac;

- The low-energy beam dump dipole (and beam dump itself) after the linac;

- The initial dipoles of the splitters at either end, since the splitter can only accommodate tunnel curvature once the beams are separated;

- Beam diagnostics at least at the linac entry and exit.

The following contingency items may also be considered for installation in this straight, depending on later design decisions and the performance of the actual machine: 
- Additional linac cavities if the design gradient cannot be achieved;

- $\quad 2^{\text {nd }}$ harmonic cavities for compensating synchrotron radiation and reducing energy spread;

- Ferrite HOM absorbers that are before and after the cavities rather than being integrated with them;

- A single-turn abort kicker system that dumps all energies at the same time.

The $2^{\text {nd }}$ harmonic cavities and single-turn abort would be placed here because this is the only place where all of the beams are going through the same beamline with a high beta function, allowing long drifts that are not possible in the FFAG lattice. So the space at two o'clock must be used carefully. The above items are not in the baseline eRHIC design but it may be prudent to leave $10 \mathrm{~m}$ or more of spare space for them.

The effect of decreasing the number of turns on the linac length, extrapolated from the previous $1.322 \mathrm{GeV}$ design is shown in Table 1. Moving up in frequency to $647 \mathrm{MHz}$ may increase the gradient and decrease the length slightly but this is a small effect.

Table 1: Number of Turns vs. Linac Length

\begin{tabular}{|l|l|l|}
\hline Number of turns $(\mathbf{N})$ & Linac energy gain $(\mathrm{GeV})$ & Linac estimated length $(\mathbf{m})$ \\
\hline 16 & 1.322 (for 21.164 total) & 123.55 \\
\hline 14 & 1.429 (for 20 total, as below) & 135.15 \\
\hline 12 & 1.667 & 155.45 \\
\hline 11 & 1.818 & 169.95 \\
\hline 10 & 2.000 & 187.35 \\
\hline 9 & 2.222 & 207.65 \\
\hline
\end{tabular}

Examining Table 1, the cases $\mathrm{N}<=9$ are not possible with the linac in a single location. The case $\mathrm{N}=10$ is marginal as there is no contingency at all once required components like the beam dump and injection dipoles are included. The cases $\mathrm{N}>=11$ are all allowed, with $\mathrm{N}=12$ and above having ample spare space. As the linac becomes shorter, it allows the splitter/combiners to be straighter, simplifying the optics and reducing their length as well.

\subsection{Number of Splitter/Combiner Lines}

Each energy in eRHIC must be separated into its own splitter/combiner line on either side of the linac. The minimum separation of these lines is $15 \mathrm{~cm}$, determined by Holger Witte's magnet design and a few lines may require larger apertures due to high energy spreads and dispersion. The baseline splitter design splits the lines initially with a single dipole, so they diverge in one particular direction from the linac (horizontally or vertically). This means that the previous design with 16 lines would take up $15 \mathrm{~cm}^{*} 16=2.4 \mathrm{~m}$, which barely fits in the RHIC tunnel vertically assuming a reasonable location for the eRHIC linac centre axis. Horizontally, there is more room above the RHIC cryostats but this would require additional vertical bends at the far end to get the lines down to where the eRHIC FFAGs are, low down and adjacent to the RHIC magnets. The large width was partially avoided in the splitter design by sending some lines onto a different plane. 
Thus, 16 splitter lines should be considered the reasonable limit, as this is the largest number for which a design has been calculated and is clearly approaching physical difficulties if it were increased any more. Having fewer splitter lines certainly simplifies the design and reduces its cost.

\subsection{Synchrotron Radiation in the FFAG Return Loops}

Assuming the beam current is kept constant at a given energy, increasing the number of turns will increase the amount of synchrotron radiation emitted simply because of greater path length while the energy range and magnetic fields stay the same. If eRHIC were composed of separate conventional recirculating lines, this increase would be roughly linear for large numbers of turns.

In the case of FFAGs, there is also the consideration that, if the number of FFAG loops remains constant at two, the energy ratio within each one will increase slightly for larger numbers of turns, approximately as $\sqrt{ } \mathrm{N}$. This will make the FFAG less optimal for synchrotron radiation. Vladimir Litvinenko calculated [eRHIC meeting, 2015-Aug-19] that the synchrotron radiation dependence should become $\mathrm{N}^{2}$ rather than just $\mathrm{N}$.

Dejan Trbojevic [eRHIC meeting, 2015-Jul-29] examined the cases for $10<=\mathrm{N}<=14$ individually, deriving an FFAG lattice and calculating the synchrotron radiation for each one. The results are summarised in Table 2.

Table 2: Number of Turns vs. Synchrotron Radiation

\begin{tabular}{|l|l|l|}
\hline Number of turns $(\mathrm{N})$ & $\begin{array}{l}\text { Synchrotron radiation for } \\
\text { I=10 } \mathrm{mA} \text { and E=20GeV (MW) }\end{array}$ & $\begin{array}{l}\text { Synchrotron radiation for } \\
\text { I=10mA and E=16GeV (MW) }\end{array}$ \\
\hline 10 & 2.76 & 1.33 \\
\hline 11 & 3.18 & 1.63 \\
\hline 12 & 4.22 & 1.75 \\
\hline 13 & 3.48 & 2.47 \\
\hline 14 & 5.15 & 2.69 \\
\hline
\end{tabular}

The values in Table 2 are fit well by an $\mathrm{N}^{2}$ law, with a doubling in power between $\mathrm{N}=10$ and $\mathrm{N}=14$ (approximately 10 $\sqrt{2}$ ). In the cases of high-energy running, where eRHIC is limited by the RF power required to balance synchrotron radiation, this would translate directly into a doubling of electron current (and luminosity) when using $\mathrm{N}=10$ as opposed to $\mathrm{N}=14$.

\subsection{Linac Total Current (HOM Damping) and BBU Instability}

Changing the number of turns does not only affect the linac length but also the total amount of current passing through it. The highest current considered for eRHIC (in the ultimate scenario) is $50 \mathrm{~mA}$ but this is current from the electron source. In an N-pass design, the beam is wrapped around through the linac a total of $2 \mathrm{~N}$ times ( $\mathrm{N}$ to accelerate, $\mathrm{N}$ to decelerate). However, due to synchrotron radiation limits, the $50 \mathrm{~mA}$ current is never used at $20 \mathrm{GeV}$. Instead, it is used at $10 \mathrm{GeV}$, which would run with $\mathrm{N} / 2$ accelerating turns. Thus the maximum total current in the eRHIC linac is $2 *(\mathrm{~N} / 2) * 50 \mathrm{~mA}=50 * \mathrm{~N} \mathrm{~mA}$. It increases linearly with $\mathrm{N}$ and the current is also the source of higherorder mode power. So the HOM power that needs to be removed from the linac per unit length also increases linearly with $\mathrm{N}$. 
An additional consideration in favour of lowering $\mathrm{N}$ is that the strength of the beam break-up instability in multi-turn ERLs is predicted to increase with a dependence somewhere between $\mathrm{N}$ and $\mathrm{N}^{2}$ depending on the phase advances of the return loops.

\subsection{Total Cost}

Changing the number of turns while keeping the total eRHIC energy constant changes two major cost items:

- Cost of the linac;

- Cost of the splitter/combiner lines.

Vladimir Litvinenko examined the scaling of these items in the most recent eRHIC bottom-up costing [eRHIC meeting, 2015-Aug-14] and arrived at the graph shown in Figure 2.

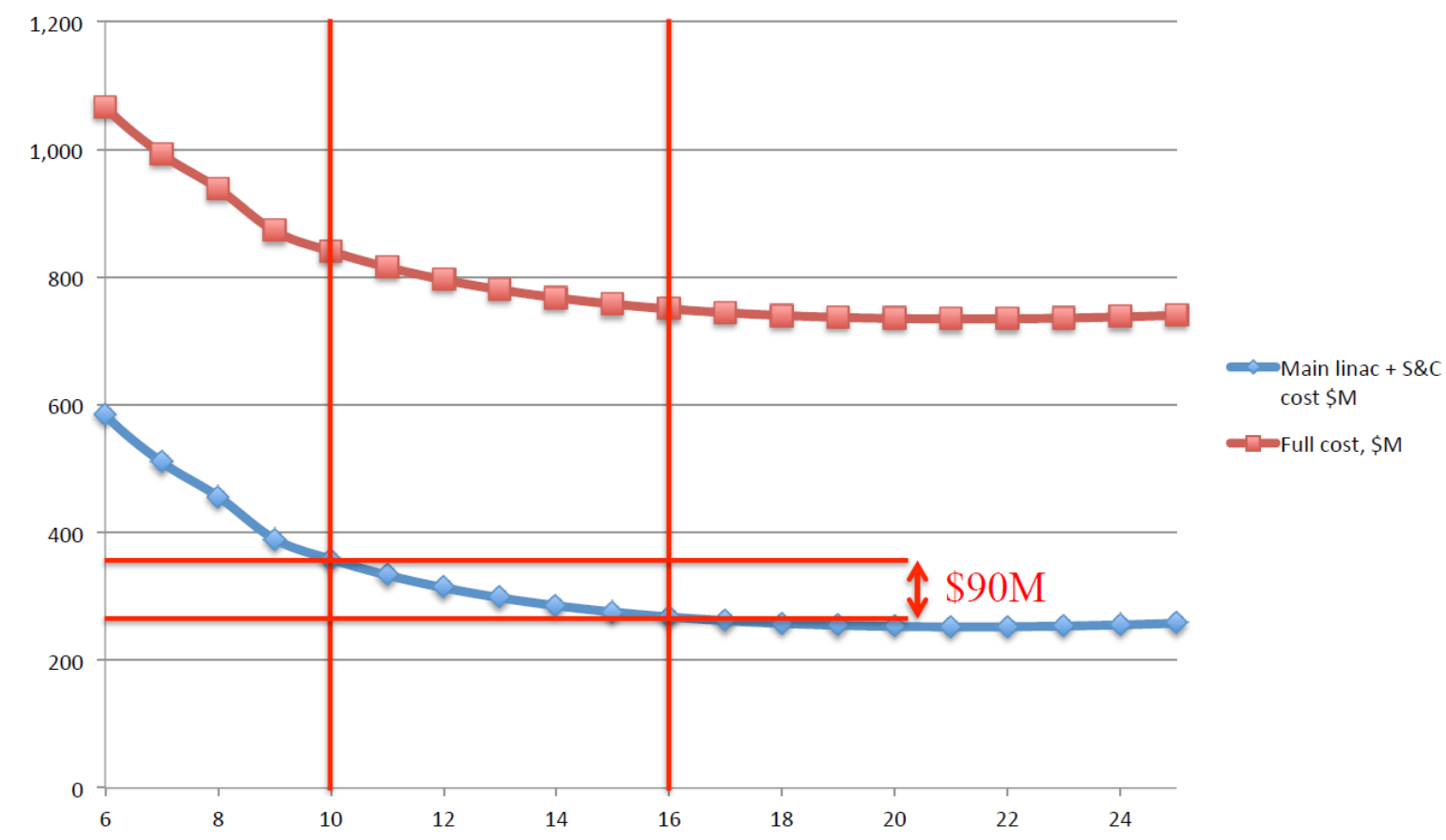

Figure 2: Cost (\$M, vertical axis) as a function of number of turns (horizontal axis).

The curve exhibits a broad optimum, with hardly any cost variation in the region from 16 to 25 turns. In this region, the additional cost of the splitter/combiner lines almost exactly cancels any cost savings from shortening the linac as $\mathrm{N}$ is increased.

The goal here is not to exactly minimise the cost, but to see if the benefits of smaller $\mathrm{N}$ explained in sections $2.2-2.4$ can be obtained for only a small increase in cost. The graph shows that reducing $\mathrm{N}$ from 16 to 10 costs an additional $\$ 90 \mathrm{M}$. Reducing $\mathrm{N}$ from 16 to 12 costs $\$ 45 \mathrm{M}$.

\section{Decision on Number of Accelerating Turns with Justification}

To summarise the constraints and preferences on total number of turns $\mathrm{N}$ from the previous sections:

- Linac length is unfeasible for $\mathrm{N}<=9$, marginal at $\mathrm{N}=10$ and $\mathrm{OK}$ for $\mathrm{N}>=11$; 
- Splitters/combiners get better as $\mathrm{N}$ is reduced with $\mathrm{N}>16$ being impractical;

- Synchrotron radiation gets better as $\mathrm{N}$ is reduced, being approximately proportional to $\mathrm{N}^{2}$;

- High-current linac effects get better as $\mathrm{N}$ is reduced, with proportionalities from $\mathrm{N}$ to $\mathrm{N}^{2}$;

- Cost is minimal at 20 turns, hardly different at 16 turns. Moving from 16 to 12 turns or from 12 to 10 each cost an additional $\$ 45 \mathrm{M}$, or $6 \%$ of total project cost.

This leaves a feasible range from $\mathrm{N}=11$ to 16 . Within this range, all considerations apart from cost get better with decreasing $\mathrm{N}$, while cost increases at an accelerating rate.

We have chosen $\mathrm{N}=12$. Reasons not to go down as far as $\mathrm{N}=11$ are the accelerating cost increase mentioned above and also the decrease in contingency space at two o'clock as the linac approaches its maximum allowed length. An additional reason relates to the energy ratios spanned by the FFAGs: a twelvefold increase can be obtained by two FFAGs of exactly 3x ratio (turns 1-3 and 4-12). However, decreasing the total range to 11 does not avoid having a $3 x$ ratio FFAG. Either it would be the low-energy loop (1-3 and 4-11) or the high energy loop would have a larger 3.67x ratio (1-2 and 3-11). Having the same factor of 3 in both FFAGs also allowed some design commonalities between them in the Nov'15 eRHIC design, such as equal cell lengths.

\section{References}

[eRHIC meeting] Presentations can be found on the main eRHIC wiki page, sorted by date: http://www.cadops.bnl.gov/eRHIC/erhicWiki/index.php/Main Page 